\title{
Multiple Failure Modes Reliability Modeling and Analysis in Crack Growth Life Based on JC Method
}

\author{
YuanTao Sun, Chao Liu, Qing Zhang, and XianRong Qin \\ School of Mechanical Engineering, Tongji University, Shanghai, China \\ Correspondence should be addressed to Qing Zhang; zhqing_tj@126.com
}

Received 3 January 2017; Accepted 14 March 2017; Published 3 April 2017

Academic Editor: Mitsuhiro Okayasu

Copyright (C) 2017 YuanTao Sun et al. This is an open access article distributed under the Creative Commons Attribution License, which permits unrestricted use, distribution, and reproduction in any medium, provided the original work is properly cited.

\begin{abstract}
The fatigue crack growth (FCG) phenomenon generally exists in large mechanical structures. Due to the influences of varied kinds of random factors, the safety evaluation of structure in FCG is under great uncertainty. In this paper, based on the reliability theory, the limit state equations of fracture failure and static strength failure were derived firstly, and the parameters in those equations were regarded as random variables that follow the normal distribution or log-normal distribution. According to the limit state equations, the JC method (equivalent normalizing method) was used to calculate the reliability indexes under the different failure modes of structure in every stress cycle. Based on the reliability indexes and correlation of the two failure modes, the joint failure probability was obtained. In the end, a specific computation example was given, and the curve of joint failure probability in multiple failure modes was used for comparison with the result of single failure mode. The results indicated that the reliability analysis based on multiple failure modes was more reasonable, and the evaluation of reliability could be obtained in fatigue crack growth process.
\end{abstract}

\section{Introduction}

It is hard to avoid cracks or other defects in mechanical structure under the current manufacturing technical condition. In the meantime, influenced by mechanical load, environment temperature, and various corrosion, fatigue cracks always occur in metal construction [1]. However, the existence of cracks leads to serious hidden danger to the safety of the structure. In previous studies, the predictions of residual life were based on the calculation of fatigue crack growth rate model, which were derived from Paris formula [2] or Forman formula [3]. Nevertheless, in many cases, the inputs of those formulas follow different distributions. In this occasion, ideal results, which can reflect the true situation, are hard to obtain by traditional certainty analyzing methods [4].

In order to solve this kind of uncertainty problems, Sankararaman et al. [5] present a methodology for uncertainty quantification and model validation in fatigue crack growth analysis. Zhang et al. [6] regard parameters $C$ and $m$ of Paris formula as random variables and then obtain the relation of those two variables through curve fitting; thus the crack growth rate can be calculated by a single variable.
What is more, the crack growth is also affected by the load; Dougherty et al. [7] develop a two-component crack growth model to treat fatigue crack growth under constant and variable amplitude loading, and the predicted crack growth agrees well with the experimental data. Liu et al. [8] put forward a new life prediction model which is based on crack growth formula, but, due to the complexity of computation, they only use the Monte Carlo method for calculation. To overcome the difficulties in the life-cycle reliability analysis of MSD, Kim et al. [9] adopt the Gaussian process (GP) response surface model for calculation, and the accuracy and advantages of the proposed method were verified by a number of experimental results and numerical examples. Zou and Yang [10] make an improvement in which the first-order second-moment method is used to calculate the reliability of structure, but the established model does not take the randomness of initial crack length into consideration, and, in practical engineering, the crack length plays an important role in the calculation of crack lifetime.

It should be noted that the current approaches for reliability analysis of structures with cracks are only based on fracture failure. However, as the crack grows, the residue cross 
section is getting smaller, which will reduce static strength. Because of the identical load and the change of crack length, fracture failure and static strength failure are correlated. Thus, only when considering the correlation of both failure modes can the failure probability be accurately obtained.

According to the above analysis and consideration, in this paper, main factors such as crack length of Paris formula were regarded as random variables. Firstly, the fracture failure reliability model and static strength failure reliability model were derived, considering the correlation of those two failure modes; then the correlation coefficient and joint failure probability expression were provided; next, the structure reliability indexes and joint failure probability were obtained by JC method. In the end, a specific computation example was given, and the curve of reliability indexes and failure probability in multiple failure modes were used for comparison with those in single failure mode.

\section{Reliability Modeling}

2.1. Reliability Modeling of Fracture Failure. Current commonly used method to calculate crack growth rate is put forward by Paris and Erdogan, and its fundamental form is

$$
\frac{d a}{d N}=C(\Delta K)^{m}
$$

where $d a / d N$ represents the crack growth rate; $C$ and $m$ denote the material variables; $\Delta K(\Delta K=Y \Delta \sigma \sqrt{\pi a}=$ $\left.Y \sqrt{\pi a}\left(\sigma_{\max }-\sigma_{\min }\right)\right)$ stands for stress intensity factor variation; $a$ is the crack length and $Y$ is shape factor. $C$ and $m$ in port machine structure were obtained through experiment [11].

Assume that the initial crack length is $a_{0}$, and under arbitrary stress cycle times $N$, the crack length became

$$
a(N)=\left[\frac{2-m}{2} C(Y \sqrt{\pi} \Delta \sigma)^{m} N+a_{0}{ }^{1-m / 2}\right]^{2 /(2-m)} \text {. }
$$

And $K_{\max }$ is given as follows:

$$
\begin{aligned}
& K_{\max }=Y \sigma_{\max } \sqrt{\pi a(N)} \\
& =Y \sigma_{\max } \sqrt{\pi}\left[\frac{2-m}{2} C(Y \sqrt{\pi} \Delta \sigma)^{m} N\right. \\
& \left.+a_{0}{ }^{1-m / 2}\right]^{1 /(2-m)} .
\end{aligned}
$$

The structure is destroyed by fracture failure when the value of $K_{\max }$ exceeds the value of fracture toughness $K_{i c}$; thus we can get the limit state equation:

$$
\begin{aligned}
g_{1}\left(C, m, K_{I C}\right)=\ln \left(\frac{K_{I C}}{K_{\max }}\right) \\
=\ln K_{I C}-\ln \left(Y \sigma_{\max } \sqrt{\pi}\right) \\
\quad-\frac{\ln \left[((2-m) / 2) C(Y \sqrt{\pi} \Delta \sigma)^{m} N+a_{0}{ }^{1-m / 2}\right]}{2-m} .
\end{aligned}
$$

Reference [12] makes a statistical analysis of distribution of $C$ and $m$, and the conclusion was that $C$ followed lognormal distribution and $m$ followed normal distribution.
Note that $K_{\max }$ is also a material property which follows lognormal distribution [13]. It should be pointed out that $Y$ can be regarded as a constant for an infinite rectangular plate; however, if the rectangular plate is finite, $Y$ is a variable which is influenced by the crack length $a$ and plate width $b$ :

$$
\begin{aligned}
Y= & 1.12-0.231\left(\frac{a}{b}\right)+10.55\left(\frac{a}{b}\right)^{2}-21.72\left(\frac{a}{b}\right)^{3} \\
& +30.39\left(\frac{a}{b}\right)^{4} .
\end{aligned}
$$

Theoretically, the value error of above formula is only $0.5 \%$, when $a / b \leq 0.6$.

2.2. Reliability Modeling of Static Strength Failure. As the fatigue crack length grows, the section modulus is getting smaller, which will cause the decrease of static strength. Based on the normal strength failure equation, the limit state equation for yield failure mode can be obtained:

$$
g_{2}(a, S)=R-\sigma=\sigma_{s} G(a)-\sigma,
$$

where $R$ represents the resistance of structure which can resist destruction; $\sigma$ denotes the fatigue loads; $\sigma_{s}$ is the yield stress; $G(a)$ stands for section modulus.

\section{Reliability Indexes Calculation by JC Method}

In this section, JC method is used to calculate the reliability indexes. JC method has many advantages: it can take the distribution of variables in limit state equations $g_{1}$ and $g_{2}$ into account, and, what is more, the points obtained by linear method based on Taylor expansion locate in the failure hypersurface. The calculation accuracy of this method can meet the practical engineering needs. It is important to note that this method requires the variables to follow normal distribution. However, variables $C$ and $m$ follow the lognormal distribution; thus we must normalize these two variables before calculation. The requirement for normalization of JC method is that the cumulative distribution function and probability density function of $X_{i}^{\prime}$ and $X_{i}$ must be the same in design point $x_{i}^{*}$.

$$
\begin{aligned}
& F_{X_{i}^{\prime}}\left(x_{i}^{*}\right)=\phi\left(\frac{x_{i}^{*}-\mu_{X_{i}^{\prime}}}{\sigma_{X_{i}^{\prime}}}\right)=F_{X_{i}}\left(x_{i}^{*}\right), \\
& f_{X_{i}^{\prime}}\left(x_{i}^{*}\right)=\frac{1}{\sigma_{X_{i}^{\prime}}} \varphi\left(\frac{x_{i}^{*}-\mu_{X_{i}^{\prime}}}{\sigma_{X_{i}^{\prime}}}\right)=f_{X_{i}}\left(x_{i}^{*}\right) .
\end{aligned}
$$

Then the mean value and standard deviation of normalized variables can be gotten.

$$
\begin{aligned}
& \mu_{X_{i}^{\prime}}=x_{i}^{*}-\phi^{-1}\left[F_{X_{i}}\left(x_{i}^{*}\right)\right] \sigma_{X_{i}^{\prime}}, \\
& \sigma_{X_{i}^{\prime}}=\frac{\varphi\left\{\phi^{-1}\left[F_{X_{i}}\left(x_{i}^{*}\right)\right]\right\}}{f_{X_{i}}\left(x_{i}^{*}\right)} .
\end{aligned}
$$


The calculation process of JC method is shown as follows $\left(\mu_{X_{i}}\right.$ and $\sigma_{X_{i}}$ are mean value and standard deviation of variables):

(1) Chose the initial design point $X^{*}$; generally we assume that $X_{i}^{*}=\mu_{X_{i}}$.

(2) Normalize variables $C$ and $m$ in design point $X^{*}$, and use the new mean value $\mu_{X_{i}^{\prime}}$ and standard deviation $\sigma_{X_{i}^{\prime}}$ to replace old ones.

(3) Calculate the direction $\operatorname{cosine} \cos \theta_{X_{i}}$ :

$$
\cos \theta_{X_{i}}=-\frac{\left(\partial g_{X}\left(x^{*}\right) / \partial X_{i}\right) \sigma_{X_{i}}}{\sqrt{\sum_{i=1}^{3}\left[\partial g_{X}\left(x^{*}\right) / \partial X_{i}\right]^{2} \sigma_{X_{i}}^{2}}} .
$$

(4) Calculate the reliability index $\beta$ :

$$
\beta=\frac{\mu_{g}}{\sigma_{g}}=\frac{g_{X}\left(x^{*}\right)+\left(\partial g_{X}\left(x^{*}\right) / \partial X_{i}\right)\left(\mu_{X_{i}}-x_{i}^{*}\right)}{\sqrt{\sum_{i=1}^{3}\left[\partial g_{X}\left(x^{*}\right) / \partial X_{i}\right]^{2} \sigma_{X_{i}}^{2}}} .
$$

(5) Obtain the new design point $X_{i}^{*}$ :

$$
X_{i}^{*}=\mu_{X_{i}}+\cos \theta_{X_{i}} \beta \sigma_{X_{i}}
$$

(6) Repeat the above steps $((2) \sim(5))$ until the relative error of $\beta$ is in the allowable error scope.

Through the JC method, we can obtain the reliability indexes of $g_{1}$ and $g_{2}$.

\section{The Calculation of Failure Probability in Multiple Failure Modes}

As mentioned above, the destruction of cracked structure is the result of both fatigue crack failure and static strength failure. In this section, how to calculate the failure probability in such multiple failure modes is presented. Assuming that these two failure modes are series systems, the joint failure probability by limit state equations $g_{1}$ and $g_{2}$ can be obtained:

$$
P_{f 12}=P\left(g_{1} \leq 0 \cup g_{2} \leq 0\right) \text {. }
$$

It can be seen from formulas (4) and (6) that both failure modes are associated with crack length $a$ and the fatigue load $\sigma$; thus the approximate computation of correlation coefficient is shown in the following formula:

$$
\begin{aligned}
& \rho_{g_{1}, g_{2}} \\
& =\frac{\left.\left(\partial g_{1} / \partial a\right)\left(\partial g_{2} / \partial a\right)\right|_{\mu} V_{a}^{2}+\left.\left(\partial g_{1} / \partial \sigma\right)\left(\partial g_{2} / \partial \sigma\right)\right|_{\mu} V_{\sigma}^{2}}{V_{g_{1}} V_{g_{2}}} .
\end{aligned}
$$

Note that $\rho_{g_{1}, g_{2}}$ is the correlation coefficient of $g_{1}$ and $g_{2} ; V$ denotes variables square deviation and $\left.\right|_{\mu}$ stands for mean values.

Then use the reliability indexes $\beta_{1} / \beta_{2}$ and correlation coefficient $\rho_{g_{1}, g_{2}}$ to calculate the joint failure probability:

$$
P_{f 12}=\phi_{2}\left(-\beta_{1},-\beta_{2}, \rho_{12}\right) \text {. }
$$

Figure 1 shows the calculation process of joint failure probability.

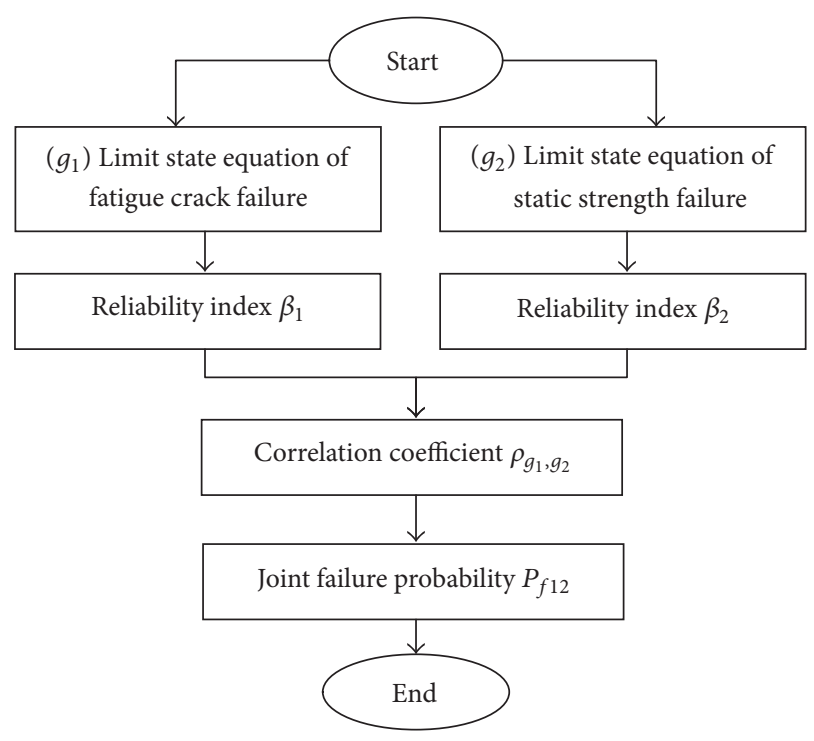

FIGURE 1: The calculation process of joint failure probability.

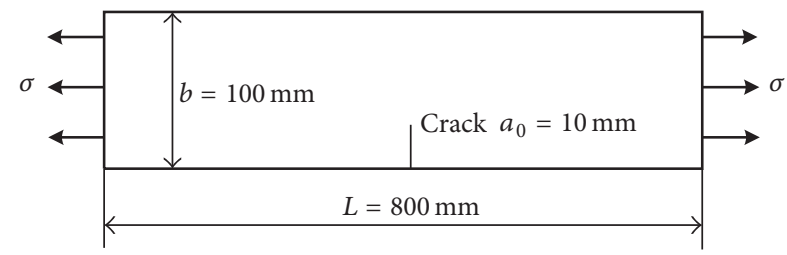

Figure 2: A single edge cracked specimen.

TABLE 1: Mean and variation coefficient of variables.

\begin{tabular}{lcc}
\hline Variables & Mean & Variation coefficient \\
\hline$\sigma$ & $90 \mathrm{MPa}$ & 0.1 \\
$\sigma_{s}$ & $235 \mathrm{MPa}$ & 0.05 \\
$C$ & $1.83 \times 10^{-13}$ & 0.01 \\
$m$ & 2.736 & 0.01 \\
$\ln K_{I C}$ & 5.2544 & 0.125 \\
$a_{0}$ & $10 \mathrm{~mm}$ & 0.01 \\
\hline
\end{tabular}

\section{Numerical Example}

In this section, an example is presented to justify the proposed method.

Example 1. Figure 2 shows a single edge cracked specimen, of which the material is Q235. The length $L=800 \mathrm{~mm}$, the width $b=100 \mathrm{~mm}$, and the thickness is $B=1 \mathrm{~mm}$. The mean and variation coefficient of variables are shown in Table 1.

According to the information given in the example, the reliability indexes of crack failure and static strength failure can be calculated. Figure 3 shows the reliability indexes in different stress cycle times.

It can be seen from Figure 3 that as stress cycle time increases, both the reliability indexes reduce. From the aspect of single failure mode, when the value of stress cycle time 


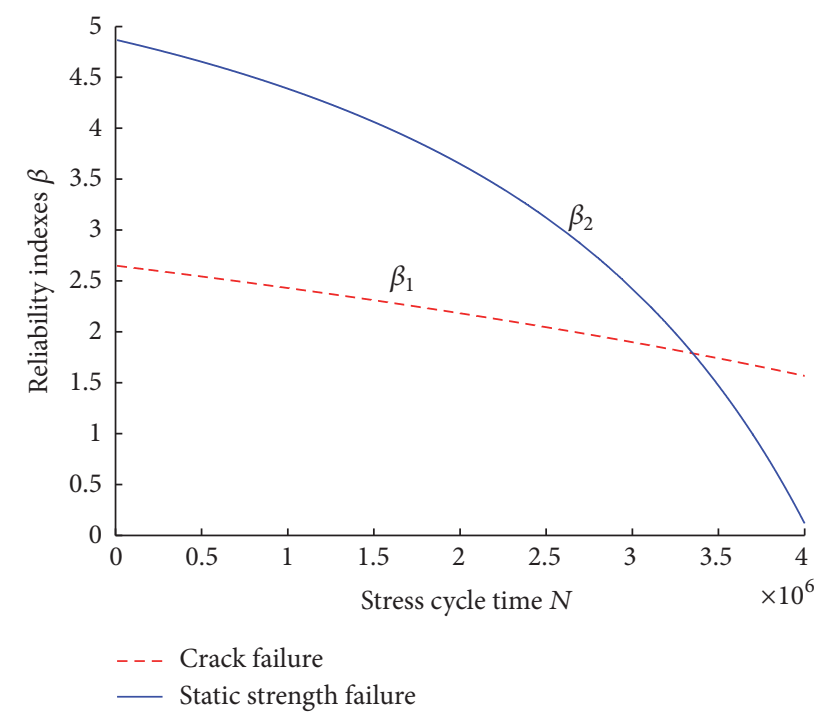

Figure 3: Reliability indexes in different stress cycle times.

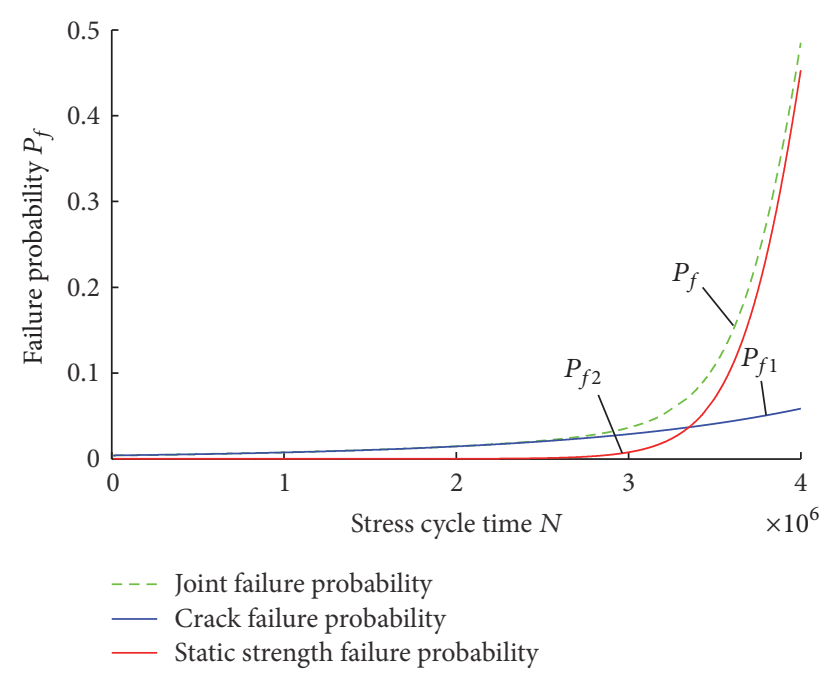

FIGURE 4: Failure probability varies with stress cycle time.

is relatively small, the destruction of structure is more likely caused by crack failure. As cycle time increases, the reliability index of static strength failure drops rapidly; while $N>3500000$, the reliability index of crack failure exceeds the static strength failure's, and structure is more likely be damaged by static strength failure.

The joint failure probability curves varying with time are shown in Figure 4. It can be seen from Figure 4 that failure probability increases with the stress cycle time; when stress cycle time $N$ reaches 3500000 , the joint failure probability increases rapidly. When $N$ is less than 3000000 , the static strength failure probability is relatively low, and the joint failure probability is close to the crack failure probability. As $N$ increases (about 3500000), the static strength failure probability increases rapidly and gets close to the joint failure probability. It should be pointed out that the joint failure probability is always higher than probability in single failure mode. Therefore, compared with the existing methods which only consider the single failure mode, this relevant theoretical method is more accurate, secure, and reliable.

\section{Summary}

The analysis of reliability of cracked structure is very important. Since the destruction of cracked structure is the result of both fatigue crack failure and static strength failure, we calculate the joint failure probability under multiple failure modes and compare it to that under single failure mode. From the results some conclusions can be drawn:

(1) Reliability indexes decrease as the stress cycle time $N$ increases. At first, the reliability index of static strength failure is bigger than that of crack failure, and it is not until $N$ increases to a certain value that the crack failure reliability index surpasses the former. This result corresponds to the engineering experiences.

(2) The correlation of crack failure and static strength failure is considered, based on which the calculation result of failure probability is obtained. The result indicates that joint failure probability is always higher than that in single failure mode, and it shows that, compared with the existing methods which only consider the single failure mode, this relevant theoretical method is more secure and reliable.

\section{Conflicts of Interest}

The authors declare that they have no conflicts of interest.

\section{Acknowledgments}

This research was supported by the National Natural Science Foundation of China (Project 51205292), the Fundamental Research Funds for the Central Universities of China (Project 20153691), the National Science and Technology Support Program (Project 2014BAF08B05, 2015BAF06B05), and Science and Technology Committee of Shanghai (Project 15DZ1161203). The authors also gratefully acknowledge the contribution and support of Shanghai Zhenhua Heavy Industries Co., Ltd.

\section{References}

[1] Y. Sun, Research on Numerical Analysis Method and Application of Crack Diagnosis for Mechanical Structure, Wuhan University of Technology, Wuhan, China, 2008.

[2] P. Paris and F. Erdogan, "A critical analysis of crack propagation laws," Journal of Basic Engineering, vol. 85, pp. 528-533, 1963.

[3] R. G. Forman, V. E. Kearney, and R. M. Engle, "Numerical analysis of crack propagation in a cyclic-loaded structure," Journal of Basic Engineering, vol. 89, pp. 459-465, 1967.

[4] X. Zhang and W. Zhao, "Reliability analysis based on residual strength and crack growth life model," Journal of Shenyang Institute of Aeronautical Engineering, vol. 5, pp. 17-21, 2009.

[5] S. Sankararaman, Y. Ling, and S. Mahadevan, "Uncertainty quantification and model validation of fatigue crack growth 
prediction," Engineering Fracture Mechanics, vol. 78, no. 7, pp. 1487-1504, 2011.

[6] H. Zhang, J. Chen, and K. Huang, "A method for estimating fatigue crack growth reliability using fatigue crack growth data," Mechanical Science and Technology, vol. 22, no. 3, pp. 384-385, 2003.

[7] D. J. Dougherty, A. D. Koning, and B. M. Hillberry, "Modeling high crack growth rates under variable amplitude loading," in Advances in Fatigue Lifetime Predictive Techniques, pp. 214-233, ASTM International, West Conshohocken, Pa, USA, 1992.

[8] Y. Liu, Y. Lv, and J. Huang, "Statistical correlation of material constants and fatigue life prediction of fatigue crack growth formula," Journal of Wuhan University of Technology, vol. 6, pp. 870-872, 2004.

[9] J.-H. Kim, T. Chau-Dinh, G. Zi, W. W. Lee, and J. S. Kong, "Probabilistic fatigue integrity assessment in multiple crack growth analysis associated with equivalent initial flaw and material variability," Engineering Fracture Mechanics, vol. 156, pp. 182-196, 2016.

[10] X. Zou and J. Yang, "JC method for reliability analysis of fatigue crack growth," in Proceedings of the 14th National Conference on Structural Engineering, vol. 4, pp. 555-558, 2005.

[11] Y. Sun, Research on the Application of Paris Formula in the Diagnosis of Structural Cracks, Wuhan University of Technology, Wuhan, China, 2005.

[12] J. Zhang and B. Ma, "Monte Carlo simulation of fatigue crack growth rate da/dN," Journal of Astronautics, vol. 1, pp. 29-38, 1987.

[13] C. Dong and R. Xia, "Fatigue crack growth life prediction model," Structure \& Environment Engineering, vol. 4, pp. 13-18, 1994. 


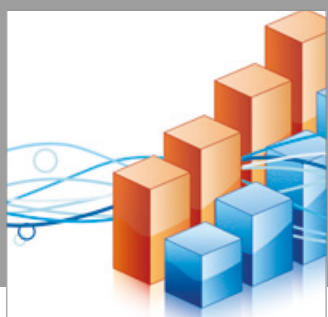

Advances in

Operations Research

vatersals

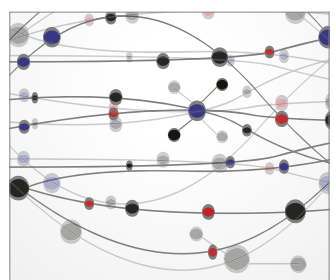

\section{The Scientific} World Journal
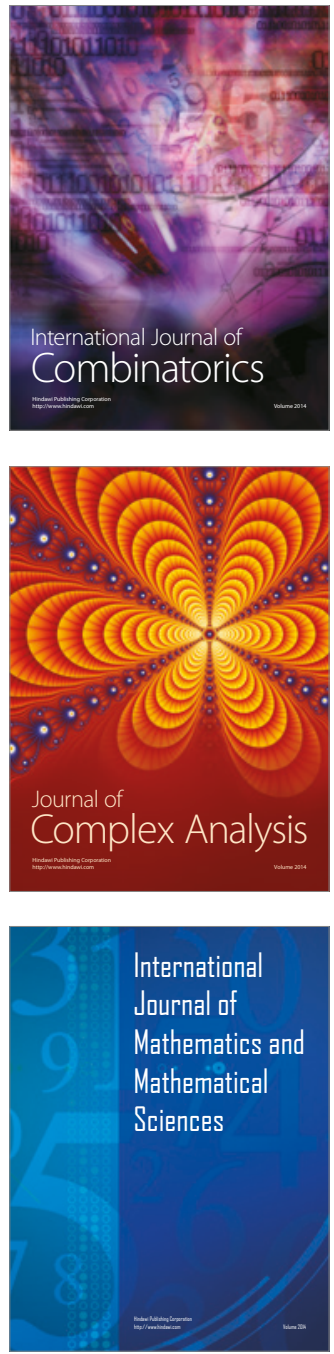
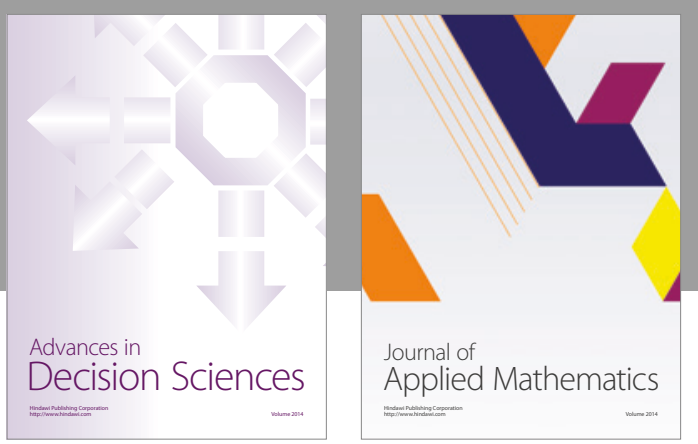

Algebra

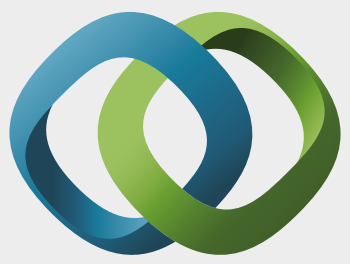

\section{Hindawi}

Submit your manuscripts at

https://www.hindawi.com
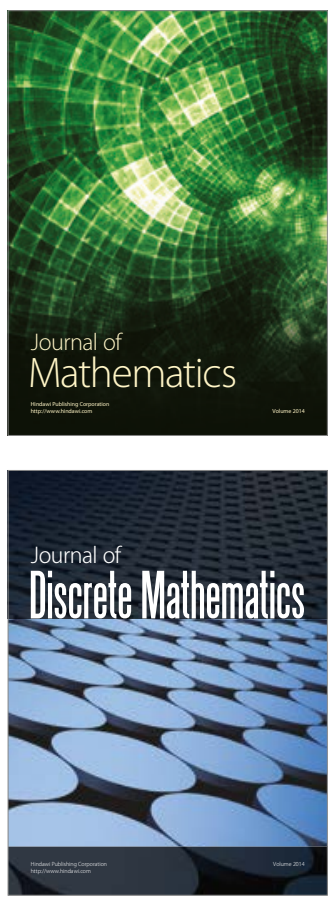

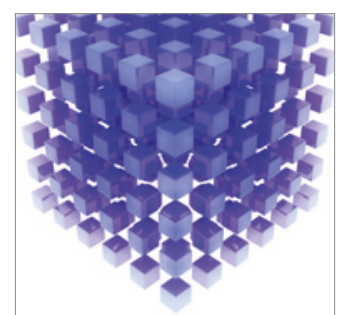

Mathematical Problems in Engineering
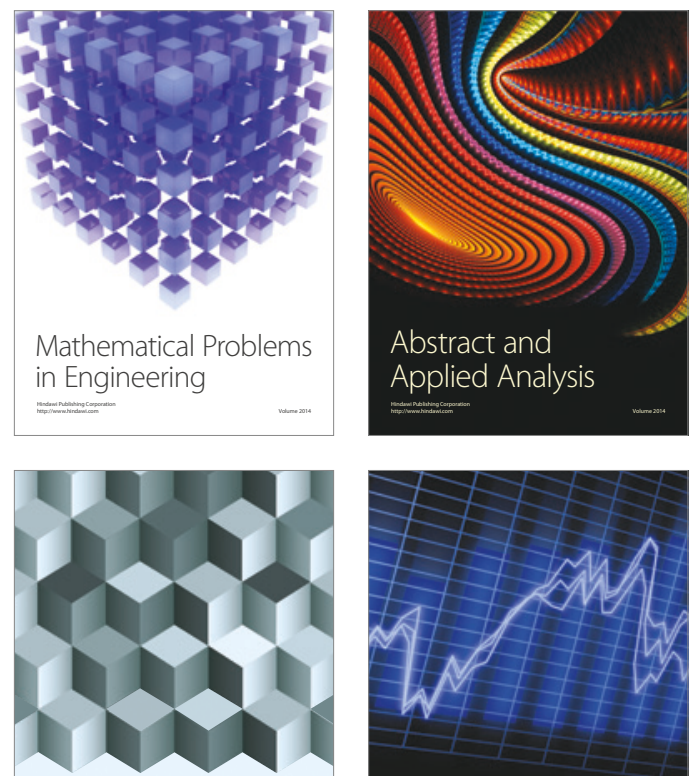

Journal of

Function Spaces

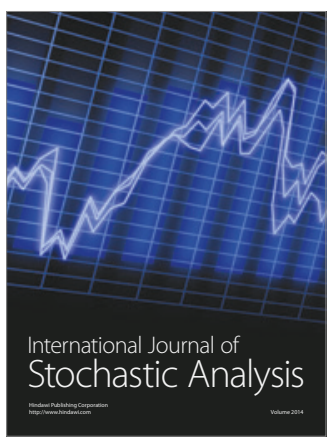

Probability and Statistics
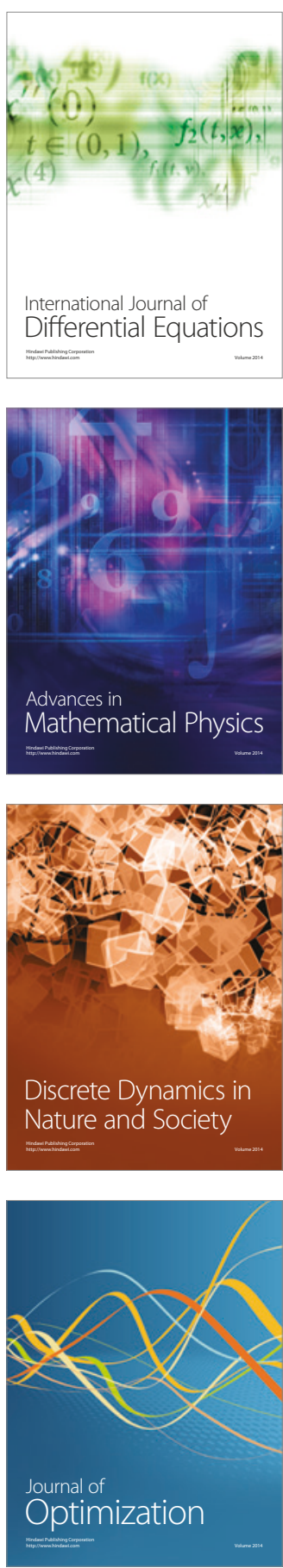